\title{
COVID-19 in patients treated with intravesical Bacillus Calmette Guerin
}

\author{
Rujittika Mungmunpuntipantip ${ }^{1 *} \odot$, Viroj Wiwanitkit ${ }^{2}$
}

\section{Dear Editor,}

We would like to share ideas on the publication "Investigation of the frequency of COVID-19 in patients treated with intravesical BCG". Karabay et al. concluded that "Intravesical BCG administration does not decrease the frequency of COVID-19 infection ${ }^{1}$." Indeed, effect of BCG on COVID19 is an interesting issue. While some authors show that BCG might be useful, others present totally discordant ideas. A common consideration on any report of the BCG effect on COVID-19 is the confounding factor. Theoretically, by molecular mechanism via tertiary lymphoid structure (TLS) organogenesis ${ }^{2,3}$, BCG might be useful. Trained immunity might occur after BCG vaccination. However, it is necessary to allow a period for immune training. From the observation by Karabay et al. ${ }^{1}$, it might be interesting to assess time effect. Additionally, many confounding factors can affect the observed frequency of COVID-194. In the report by Karabay et al. ${ }^{1}$, the number of subjects are also few. A larger study might give different results.

\section{AUTHORS" CONTRIBUTIONS}

RM: Conceptualization, Data curation, Formal analysis, Funding acquisition, Investigation, Methodology, Project administration, Resources, Software, Supervision, Validation, Visualization, Writing - original draft, Writing - review \& editing. WW: Conceptualization, Data curation, Formal analysis, Funding acquisition, Investigation, Methodology, Project administration, Resources, Software, Supervision, Validation, Visualization, Writing - original draft, Writing - review \& editing.

\section{REFERENCES}

1. Karabay O, Köse O, Tocoglu A, Uysal B, Dheir H, Yaylaci S, et al. Investigation of the frequency of COVID-19 in patients treated with intravesical BCG. Rev Assoc Med Bras (1992). 2020;66Suppl 2(Suppl 2):91-5. https://doi.org/10.1590/1806-9282.66.S2.91

2. O'Neill LAJ, Netea MG. BCG-induced trained immunity: can it offer protection against COVID-19? Nat Rev Immunol. 2020;20(6):335-7. https://doi.org/10.1038/s41577-020-0337-y
3. Koti M, Morales A, Graham CH, Siemens DR. BCG vaccine and COVID-19: implications for infection prophylaxis and cancer immunotherapy. J Immunother Cancer. 2020;8(2):e001119. https://doi.org/10.1136/jitc-2020-001119

4. Wiwanitkit V. COVID-19 death and BCG vaccination. Tuberc Respir Dis (Seoul). 2021;84(1):84. https://doi.org/10.4046/ trd.2020.0115

\footnotetext{
'Private Academic Consultant - Bangkok, Thailand.

${ }^{2}$ Dr. D. Y. Patil University - Pune, India.

*Corresponding author: rujittika@gmail.com

Conflicts of interest: the authors declare there is no conflicts of interest. Funding: none.

Received on June 11, 2021. Accepted on July 14, 2021.
} 